\title{
E47 upregulates $\Delta$ Np63a to promote growth of squamous cell carcinoma
}

\author{
Jing $\mathrm{Xu}^{1}$, Fengtian $\mathrm{Li}^{1}, \mathrm{Ya} \mathrm{Gao}{ }^{1}$, Rongtian Guo', Liangping Ding ${ }^{1}$, Mengyuan Fu', Yong Yi ${ }^{1}, \mathrm{Hu}$ Chen $\mathbb{D}^{1}$, \\ Zhi-Xiong Jim Xiao' and Mengmeng Niu (i)
}

\begin{abstract}
Targeted therapy has greatly improved both survival and prognosis of cancer patients. However, while therapeutic treatment of adenocarcinoma has been advanced greatly, progress in treatment of squamous cell carcinoma (SCC) has been slow and ineffective. Therefore, it is of great importance to decipher mechanisms and identify new drug targets involved in squamous cell carcinoma development. In this study, we demonstrate that E47 plays the distinctive and opposite roles on cell proliferation in adenocarcinoma and squamous cell carcinoma. While E47 suppresses cell proliferation in adenocarcinoma cells, it functions as a oncoprotein to promote cell proliferation and tumor growth of squamous cell carcinoma. Mechanistically, we show that E47 can directly bind to the promoter and transactivate $\triangle N p 63$ gene expression in squamous cell carcinoma cells, resulting in upregulation of cyclins D1/E1 and downregulation of $\mathrm{p} 21$, and thereby promoting cell proliferation and tumor growth. We further show that expression of E2A (E12/E47) is positively correlated with p63 and that high expression of E2A is associated with poor outcomes in clinical samples of squamous cell carcinoma. These results highlight that the E47- $\triangle \mathrm{Np} 63 \mathrm{a}$ axis may be potential therapeutic targets for treatment of squamous cell carcinoma.
\end{abstract}

\section{Introduction}

Lung cancer is the leading cause of cancer-related death worldwide. Non-small cell lung cancer (NSCLC) accounts for $85 \%$ of all cases of lung cancers ${ }^{1,2}$. NSCLC is mainly comprised of adenocarcinoma and squamous cell carcinoma (SCC), both of which exhibit unique characters including tumor origins, causation, and genomic alterations $^{3-5}$. Activating mutations in epidermal growth factor receptor (EGFR) and anaplastic lymphoma kinase (ALK) rearrangement are the major driving force for lung adenocarcinoma while most squamous cell carcinomas display somatic mutation of $\mathrm{p} 53^{4,6-8}$. In recent years, EGFRtargeted and ALK-targeted therapies have been developed and have greatly improved clinical outcomes of patients

\footnotetext{
Correspondence: Mengmeng Niu (niumm@scu.edu.cn)

${ }^{1}$ Center of Growth, Metabolism and Aging, Key Laboratory of Bio-Resource and Eco-Environment, Ministry of Education, College of Life Sciences, Sichuan

University, Chengdu, China

These authors contributed equally: Jing Xu, Fengtian Li

Edited by E. Candi
}

with lung adenocarcinoma ${ }^{9-11}$. However, there are lack of specific targeted therapies for treatment of squamous cell carcinoma. Patients with squamous cell carcinoma, including lung squamous cell carcinoma (LUSC) and head and neck squamous cell carcinoma (HNSCC), suffer significantly poorer overall survival than those with adenocarcinoma ${ }^{12-19}$. Therefore, it is of great importance to decipher unique mechanisms and identify drug targets involved in the development of squamous cell carcinoma.

It has been documented that p63, a member of p53 family, is frequently overexpressed in squamous cell carcinoma $^{4}$. p63 consists of TAp63 and $\Delta$ Np63 isoforms due to two alternative transcription start sites at the $\mathrm{N}$ terminus. Furthermore, alternative splicing at the $C$ terminus gives rise to five different $C$ terminal isoforms $(\alpha, \beta, \gamma, \delta$, and $\varepsilon$ ) of $\mathrm{p} 63^{20-23}$. Among these p63 protein isoforms, $\Delta \mathrm{Np} 63 \alpha$ is the major p63 protein isoform specifically expressed in epithelial cells. $\Delta \mathrm{Np} 63 \alpha$ can function as a proto-oncogene in promoting cancer cell proliferation and survival ${ }^{24-27}$. It has been well documented that

\section{(c) The Author(s) 2021, corrected publication 2022}

(c) OPen Access This article is licensed under a Creative Commons Attribution 4.0 International License, which permits use, sharing, adaptation, distribution and reproduction cc) in any medium or format, as long as you give appropriate credit to the original author(s) and the source, provide a link to the Creative Commons license, and indicate if changes were made. The images or other third party material in this article are included in the article's Creative Commons license, unless indicated otherwise in a credit line to the material. If material is not included in the article's Creative Commons license and your intended use is not permitted by statutory regulation or exceeds the permitted use, you will need to obtain permission directly from the copyright holder. To view a copy of this license, visit http://creativecommons.org/licenses/by/4.0/. 
$\Delta \mathrm{Np} 63 \alpha$ can directly bind to the promoter of CDKNIA (also known as p21CIP1) to inhibit its transcription, thereby promoting cell cycle progression and suppression of cell apoptosis ${ }^{28-30} . \Delta \mathrm{Np} 63 \alpha$ can also promote tumor growth by downregulation expression of growthsuppressing genes including PUMA and MIC- $1^{31,32}$, and upregulation expression of growth-promoting genes including cyclins D1 and E1 (CCND1, CCNE1) ${ }^{33,34}$. In addition, p63 can form hetero oligomers with p53 and inhibits its transcription function ${ }^{35}$.

The TCF3 gene, also called E2A, encodes two alternative splicing variants, E47 and E12, both of which are members of basic helix-loop-helix (bHLH) transcription factor ${ }^{36}$. E47 can form homodimer or heterodimer to bind to the canonical E-Box response element (CANNTG) and regulate transcription of the target genes ${ }^{37}$. It has been shown that E47 plays a critical role in promoting B-cell lymphopoiesis, T-cell development, myogenesis, and cell proliferation ${ }^{38-40}$. On the other hand, it has been reported that E47 can function as a growth suppresser in adenocarcinoma ${ }^{41,42}$. Under that circumstances, E47 can downregulate Myc protein expression and upregulate expression of p27 or p21 protein to inhibit cell-cycle progression and promote senescence $^{43,44}$. However, the role of E47 in squamous cell carcinoma is largely unclear. In this study, we demonstrate that E47 plays the inhibitory role on cell proliferation in adenocarcinoma. By sharp contrast, E47 functions as a oncoprotein to promote growth of squamous cell carcinoma through directly transactivating $\Delta \mathrm{Np} 63 \alpha$ expression.

\section{Results}

E47 plays distinctive and opposite roles in regulation cell proliferation of adenocarcinoma and squamous cell carcinoma

It has been reported that E47 inhibits cell proliferation in adenocarcinoma, while its roles in SCC are largely unknown. In this study, we aimed to examine the roles of E47 on cell proliferation and tumor growth of SCC. With this regard, we first examined E47 protein expression in cancers utilizing Oncomine database. As shown in Fig. 1A, B, E47 was lowly expressed in adenocarcinoma of lung and esophagus, whereas E47 was highly expressed in SCC of lung and esophagus. Consistently, the expression of E47 in adenocarcinoma A549 cells was lower than that in SCC FaDu and H292 cells (Fig. S1A). Moreover, ectopic expression of E47 inhibited cell proliferation in adenocarcinoma A549 and H1299 cells, as evidenced by MTS assays and colony formation assays (Fig. 1C, D), while ectopic expression of E47 promoted cell proliferation in SCC H292, FaDu, and KYSE150 cells (Fig. 1E, F and Fig. S1B). Unlike wild-type E47, E47 ${ }^{\text {A592N/1596D }}$ mutant $\left(\mathrm{E} 47^{\mathrm{DM}}\right)$ defective in its transcriptional activity ${ }^{45}$, had little effects on cell proliferation (Fig. $1 \mathrm{C}-\mathrm{F}$ and Fig. S1B). These data indicate that E47 plays the opposite roles on cell proliferation in adenocarcinoma and SCC and that E47 functions as a oncoprotein in SCC, which is dependent on its transcriptional activity. In keeping with this notion, E47 inhibited expression of Cyclin E1 and Cyclin D1 and upregulated expression of cell-cycle inhibitor p21 in adenocarcinoma A549 and H1299 cells (Fig. 1G). By sharp contrast, E47 significantly upregulated expression of Cyclin E1 and Cyclin D1 and suppressed expression of p21 in SCC H292 and FaDu cells (Fig. 1H).

We then investigated the molecular basis by which $\mathrm{E} 47$ promotes cell proliferation in SCC. It has been documented that overexpression of p63 is a typical feature of $\mathrm{SCC}^{4}$. We therefore asked whether $\Delta \mathrm{Np} 63 \alpha$ plays a role in E47-mediated stimulation of SCC cell proliferation. As shown in Fig. 1I and Fig. S1C, ectopic expression of E47 significantly upregulated $\Delta \mathrm{Np} 63 \alpha$ expression, concomitant with increased Cyclins D1/E1 expression and decreased p21 expression in H292, FaDu, and KYSE150 cells, while ectopic expression of $\mathrm{E} 47^{\mathrm{DM}}$ mutant was unable to do so. We then used shRNAs specific for E2A (E12/E47) to knockdown of E47 in H292, FaDu, and KYSE150 cells. As shown in Fig. 1J-L and Fig. S1D, E, silencing of E47 significantly reduced expression of $\Delta \mathrm{Np63 \alpha}$, concomitant with decreased Cyclins E1/D1 expression, increased $\mathrm{p} 21$ expression, and suppression of cell proliferation. Together, these results indicate that E47 significantly upregulates $\Delta \mathrm{Np} 63 \alpha$ protein expression and promotes cell proliferation in SCC cells.

\section{E47 promotes SCC cell proliferation and tumor growth via upregulation of $\Delta \mathrm{Np} 63 \mathrm{a}$ expression}

To investigate the causative role of $\Delta \mathrm{Np} 63 \alpha$ in E47induced cell proliferation, we performed the rescuing experiments. As shown in Fig. 2A, silencing of $\Delta \mathrm{Np} 63 \alpha$ completely reversed E47-induced changes expression of Cyclin E1, Cyclin D1, and p21, all of which are downstream effectors of $\Delta \mathrm{Np} 63 \alpha$. Consistently, silencing of $\Delta \mathrm{Np} 63 \alpha$ significantly rescued E47-mediated cell proliferation in H292 and FaDu cells, as evidenced by MTS assays and colony formation assays (Fig. 2B-D). In addition, ectopic expression of wild-type $\mathrm{E} 47$, but not $\mathrm{E} 47^{\mathrm{DM}}$, led to increased BrdU+ cell and S-phase population in SCC H292 cells, both of which were rescued by simultaneous silencing of $\Delta \mathrm{Np} 63$ (Fig. 2E, F and Fig. S2A). By contrast, silencing of E47 led to an increase of the BrdU+ cells in H1299 cells (Fig. S2B). These results indicate that $\Delta \mathrm{Np} 63 \alpha$ plays a causative role in E47-induced cell proliferation of SCC.

To further substantiate this conclusion, we examined the role of $\mathrm{E} 47-\Delta \mathrm{Np} 63 \alpha$ in tumor growth in vivo. As shown in Fig. 2G, H, ectopic expression of E47, but not $\mathrm{E} 47^{\mathrm{DM}}$, dramatically promoted tumor growth in $\mathrm{H} 292$ xenograft mouse model. Notably, silencing of $\Delta \mathrm{Np} 63 \alpha$ completely rescued E47-induced tumor growth (Fig. 2G, H). Furthermore, Flag-E47-expressing tumors exhibited 


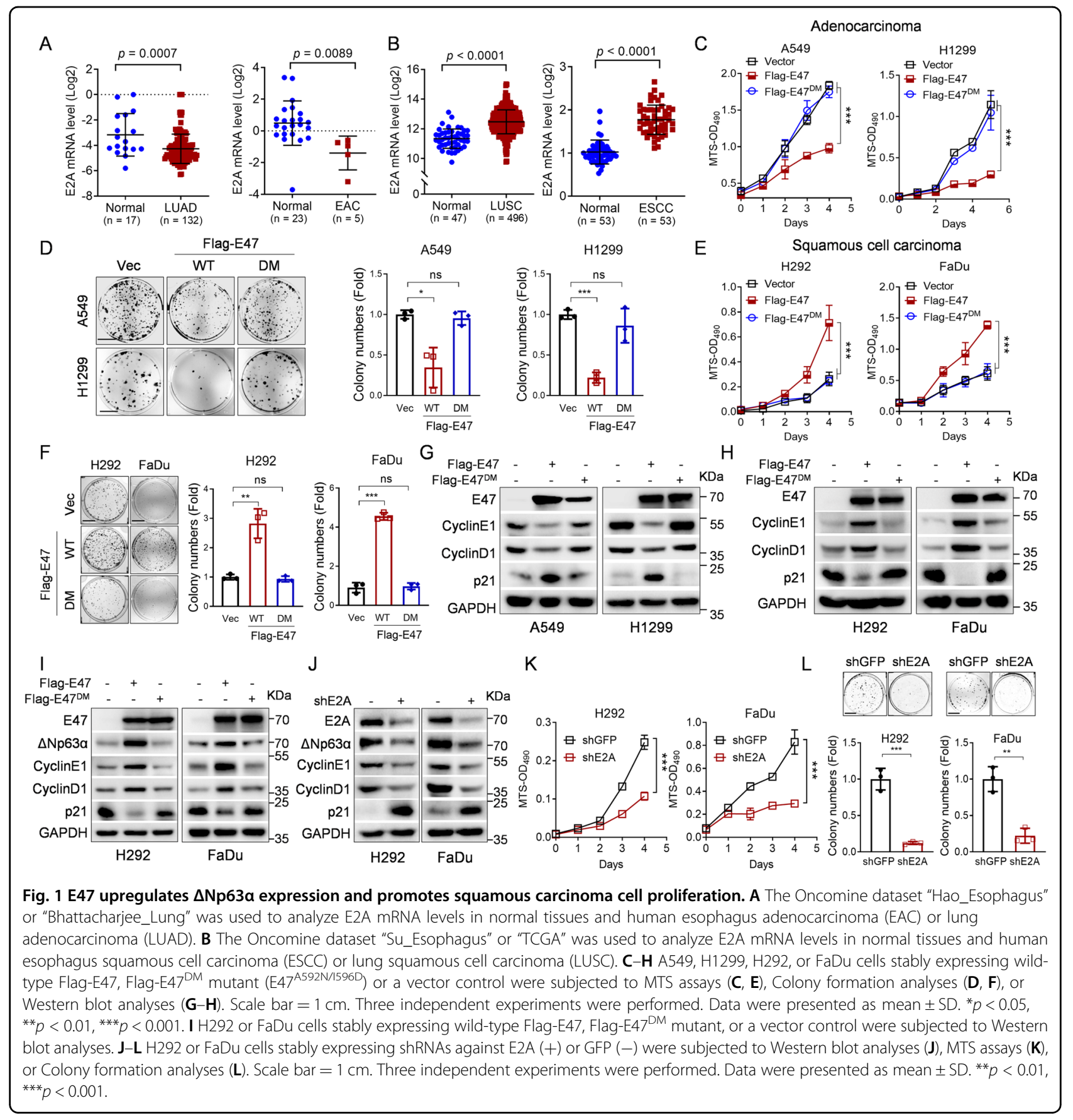

significant increased Ki67+ cells and decreased cleaved caspase-3 $+(\mathrm{CC} 3+)$ cells, all of which were rescued by silencing of $\Delta \mathrm{Np} 63 \alpha$ (Fig. 2I), indicating that E47mediated upregulation of $\Delta \mathrm{Np} 63 \alpha$ consequently facilitates tumor growth through increased cell proliferation and reduced apoptotic cell death. Together, these results demonstrate that $\Delta \mathrm{Np} 63 \alpha$ is an essential downstream effector of E47 in promoting cell proliferation and tumor growth in SCC.

\section{$\Delta$ Np63 is a direct E47 transcriptional target}

Since our aforementioned results show that E47mediated upregulation of $\Delta \mathrm{Np} 63 \alpha$ expression is dependent on its transcriptional activity, we then investigated whether $E 47$ can regulate $\Delta \mathrm{Np} 63 \alpha$ gene transcription. As shown in Fig. 3A, silencing of E2A significantly reduced steady-state $\triangle \mathrm{Np} 63 \mathrm{mRNA}$ levels, concomitant with upregulation of steady-state p21 (CDKN1A) mRNA levels and downregulation of steady-state mRNA levels of 

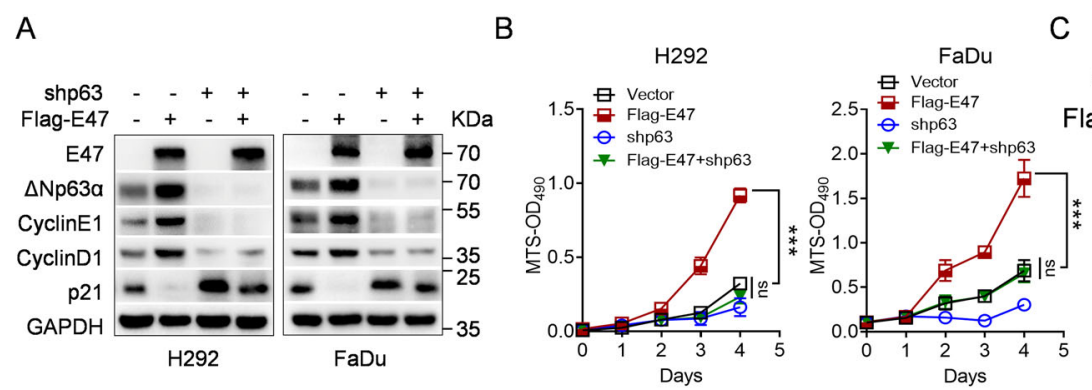

C

D

E

$\mathrm{F}$

Days
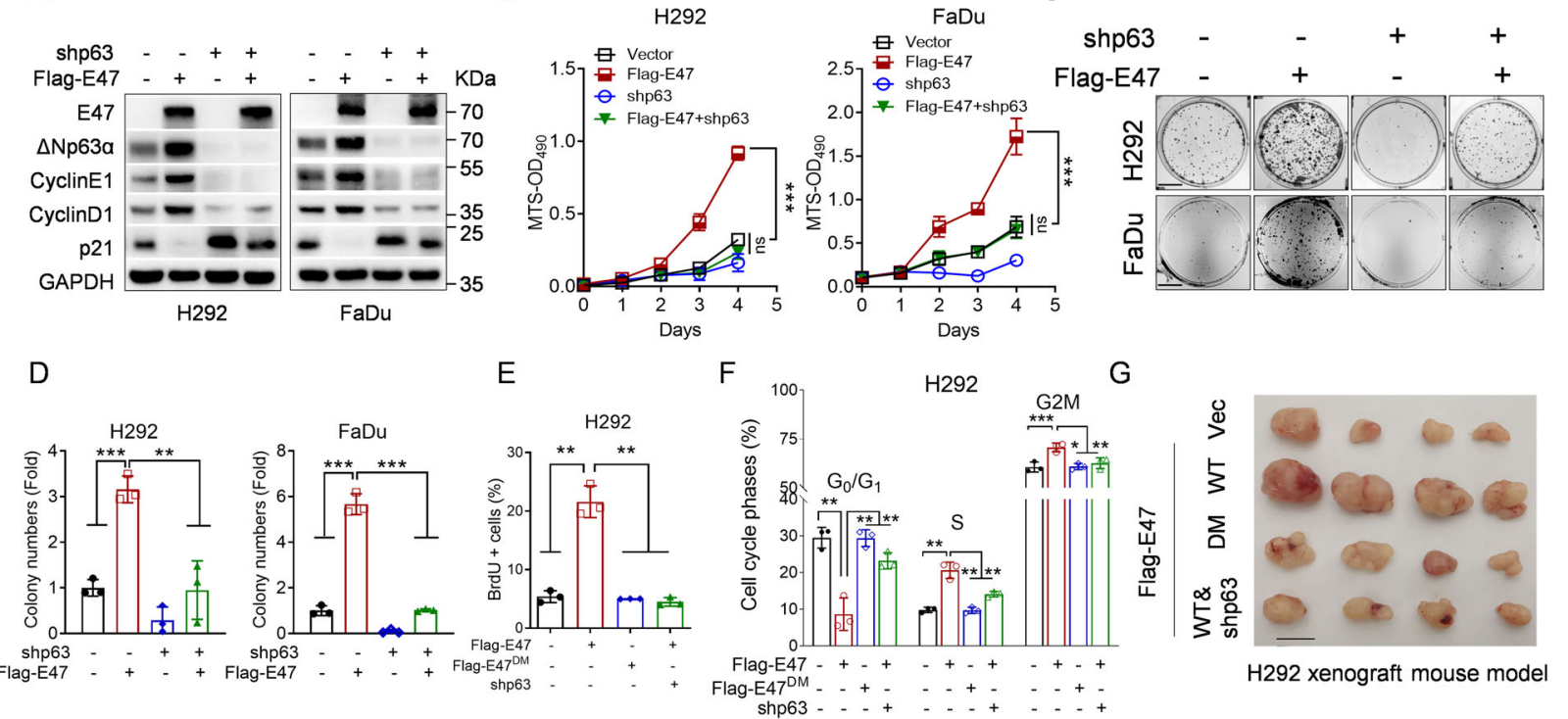

G

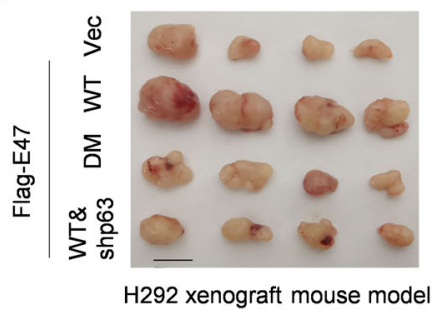

$\mathrm{H}$

I
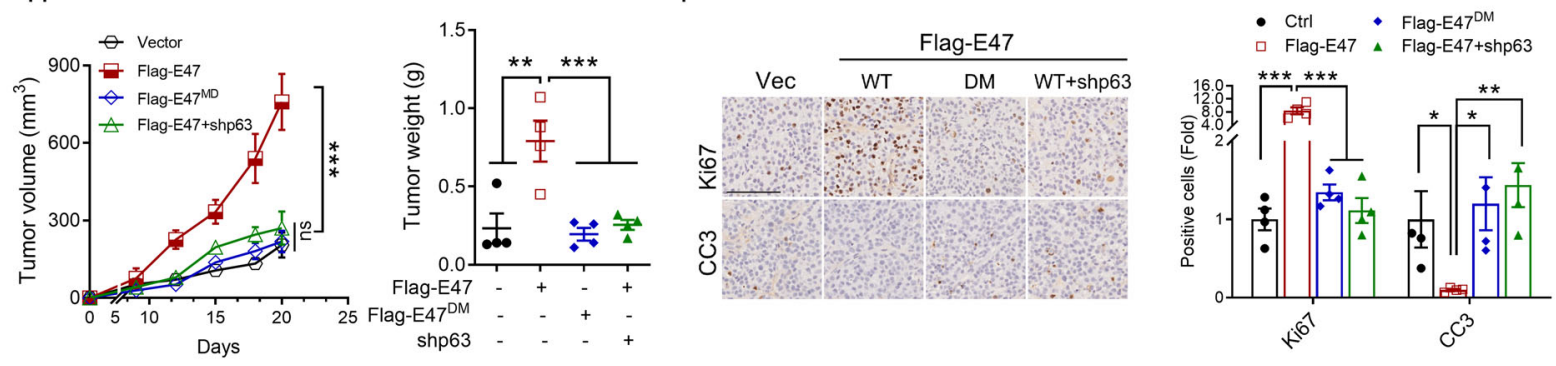

Fig. 2 Knockdown of $\Delta$ Np63a blocks E47-mediated cell proliferation and tumor growth in vitro and in vivo. A-D H292 or FaDu cells stably expressing either Flag-E47 or shp63, or both were subjected to Western blot analyses (A), MTS assays (B), or Colony formation analyses (C, D). Scale $\mathrm{bar}=1 \mathrm{~cm}$. Three independent experiments were performed. Data were presented as mean \pm SD. ${ }^{* *} p<0.01,{ }^{* * *} p<0.001$. E, $\mathbf{F} H 292$ cells stably expressing either Flag-E47 (wild-type or DM mutant) or shp63, or both were subjected to BrdU or PI staining and FACS analyses. Three independent experiments were performed. Data were presented as mean \pm SD. ${ }^{*} p<0.05,{ }^{* *} p<0.01,{ }^{* *} p<0.001 . \mathbf{G}, \mathbf{H ~ H} 292$ cells $\left(1 \times 10^{6}\right)$ stable cells were subcutaneously injected into BALB/C nude mice ( $n=4$ /group). Tumor sizes were measured every $2-3$ days. Mice were euthanized by day 20 after inoculation and individual tumor weight was measured. Tumor photos, tumor volume, and tumor weight were presented. Scale bar $=1 \mathrm{~cm}$. Data were presented as means \pm SEM. ${ }^{* *} p<0.01,{ }^{* * *} p<0.001$. I Tumors were fixed, embedded in paraffin, sectioned, and IHC was performed for Ki67 and cleaved caspase-3 (CC3). Scale bar $=100 \mu \mathrm{m}$. Data were presented as means \pm SEM. ${ }^{*} p<0.05,{ }^{* *} p<0.01,{ }^{* * *} p<0.001$.

Cyclin E1 (CCNE1) and Cyclin D1 (CCND1). Conversely, ectopic expression of E47 dramatically upregulated steady-state $\triangle \mathrm{Np} 63 \mathrm{mRNA}$ levels, concomitant with the changes of steady-state mRNA levels of Cyclin E1, Cyclin D1, and p21 in H292 and FaDu cells (Fig. 3B). Unlike wild-type E47, E47 ${ }^{\mathrm{DM}}$ mutant failed to do that (Fig. 3B). Furthermore, silencing of $\Delta \mathrm{Np} 63 \alpha$ significantly rescued E47-mediated changes of steady-state mRNA levels of Cyclin E1, Cyclin D1 and p21 in H292 and FaDu cells (Fig. 3C). Together, these results indicate that E47 upregulates $\Delta \mathrm{Np} 63$ gene transcription, which, in turn, regulates transcription of cyclins D1/E1 and p21.

To examine whether $\Delta \mathrm{Np} 63 \alpha$ is a direct E47 transcriptional target, we performed ChIP assay. As shown in Fig. 3D, the $\triangle \mathrm{Np} 63$ promoter sequence was analyzed by JASPER (http://jaspar.genereg.net), which revealed three putative E47 binding sites, including P1 (-2244 to $-2235) ; \mathrm{P} 2(-1665$ to -1656$)$ and P3 ( -828 to -819$)$ that match to the consensus sequence of E47 binding sites CANNTG ${ }^{37}$. Data from ChIP experiments showed that E47 directly bound P1 and P2 but not P3 sites in H292 cells (Fig. 3E, F). In addition, luciferase reporter assays showed that ectopic expression of E47 significantly enhanced $\Delta$ Np63-Gluc reporter $(-2000 \sim-1)$ activities in a dose-dependent manner, but failed to enhance the activities of $\Delta$ Np63-Gluc reporter with a deletion of P2 site ( $\Delta-1665$ to -1656$)$ (Fig. 3G). These results demonstrate that $\mathrm{E} 47$ can directly bind to the promoter and transactivate $\Delta \mathrm{Np} 63$ gene expression in squamous cell carcinoma cells.

We further examined whether E47 could bind to p63 promoter and transactivate p63 gene expression in 


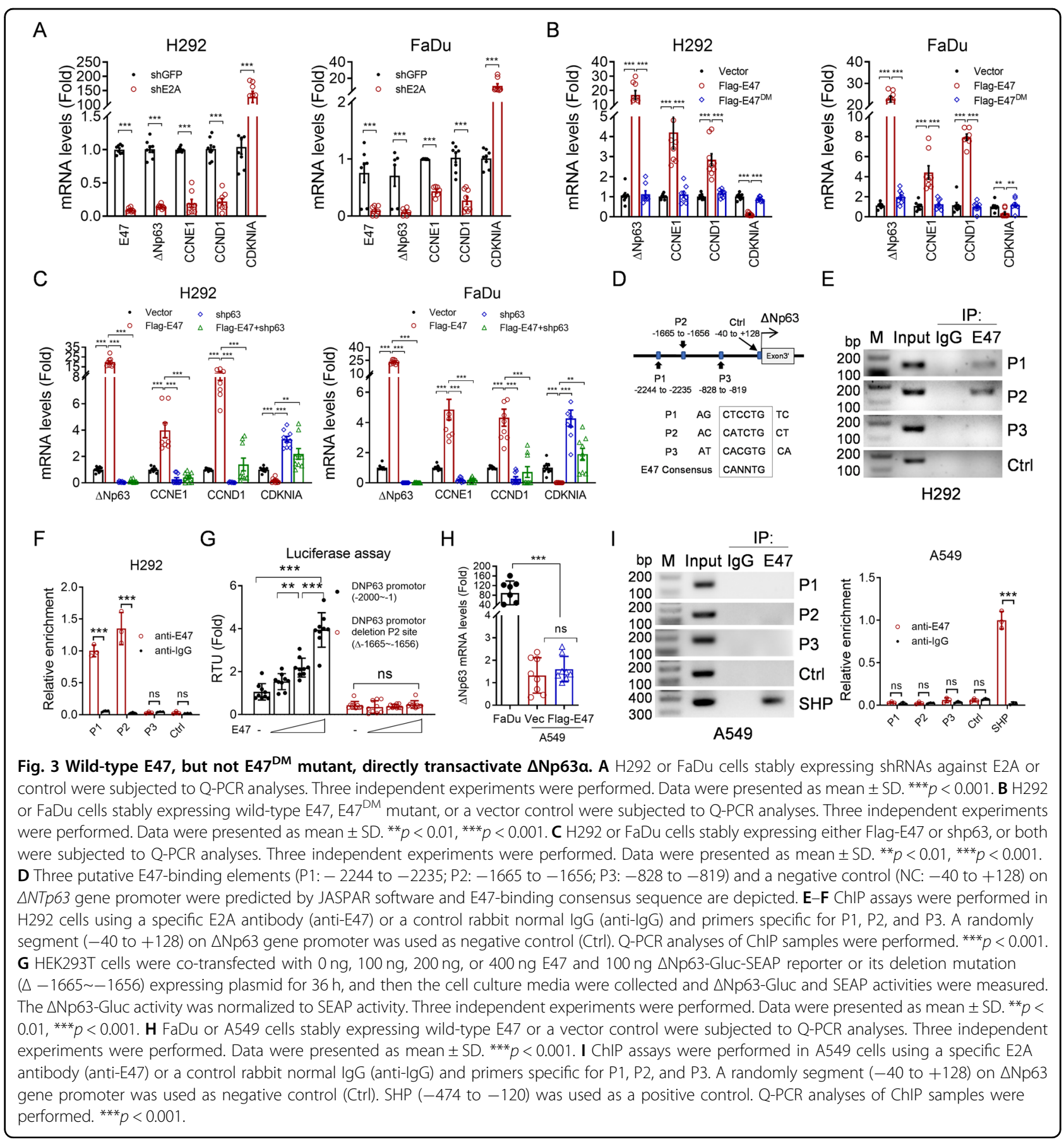

adenocarcinoma cells. As shown in Fig. 3H, ectopic expression of $\mathrm{E} 47$ was unable to upregulate steady-state $\triangle$ Np63 mRNA levels in A549 cells. In addition, while E47 bound small heterodimer partner (SHP), a known target of $E 47^{46}$, as expected, it failed to bind to any of three putative $\mathrm{E} 47$ binding sites on $\triangle \mathrm{Np} 63$ promoter in A549 cells (Fig. 3I). These results indicate that $\Delta \mathrm{Np} 63 \alpha$ is a direct E47 transcriptional target in SCC cells, but not in adenocarcinoma cells.
E2A is positively correlated with p63 expression in SCC and its high expression is associated with poor overall survival of SCC patients

Our results prompted us to verify the clinical relevance of the E47-p63 axis in squamous cell carcinoma. As shown in Fig. 4A, a positive correlation between E47 and $\triangle \mathrm{Np} 63$ protein levels was observed in 4 out of $6 \mathrm{SCC}$ cell lines examined. In addition, oncomine analyses showed that E2A (E12/E47) mRNA levels were positively 


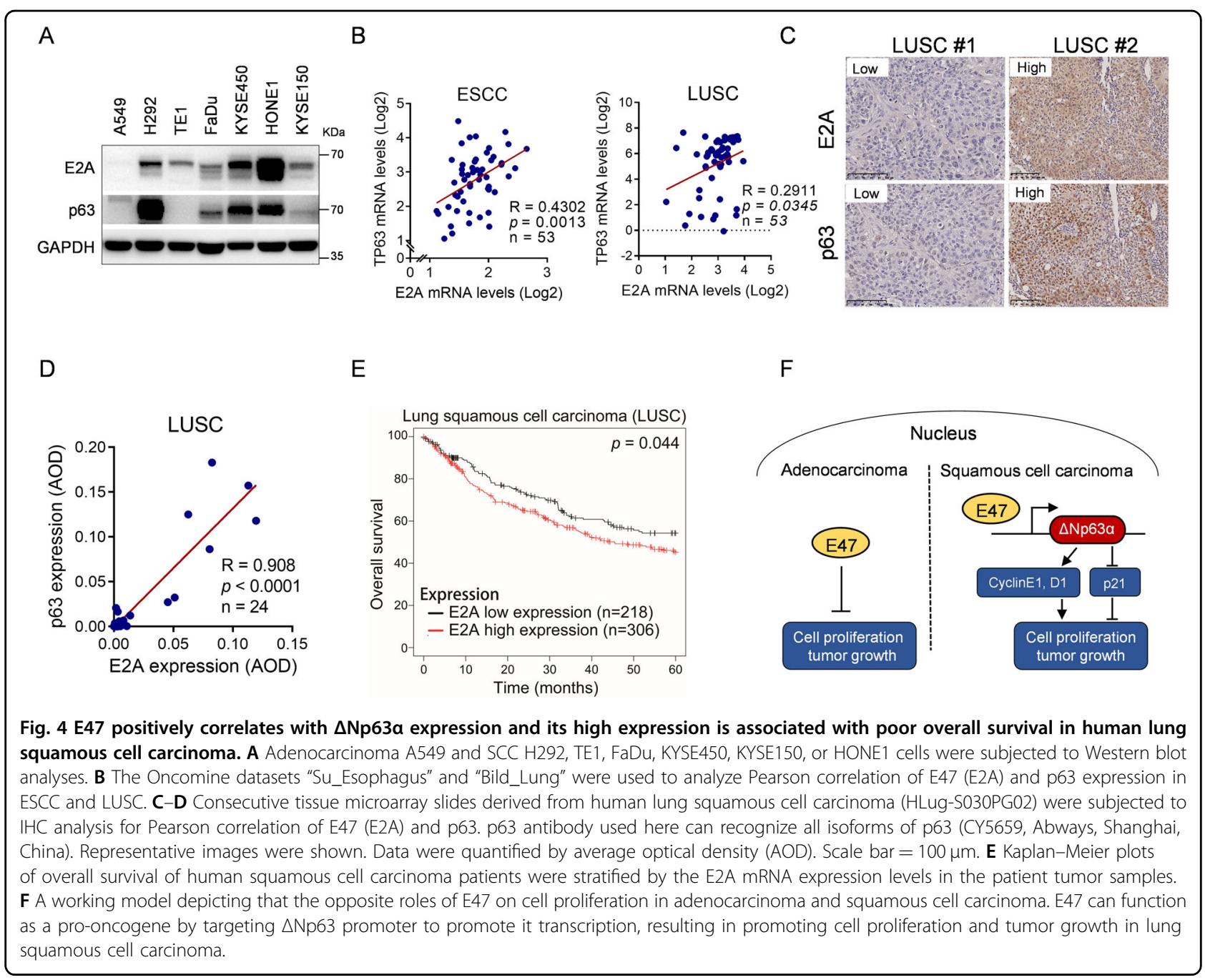

correlated with p63 mRNA levels in esophagus squamous cell carcinoma (ESCC) $(R=0.4302 ; p=0.0013)$ and in lung squamous cell carcinoma (LUSC) $(R=0.2911 ; p=$ 0.0345) (Fig. 4B). To further investigated the clinical relevance of the E47-p63 axis, we performed immunohistochemical (IHC) analyses of human LUSC tissue microarrays $(n=24)$. As shown in Fig. $4 \mathrm{C}$, D, a significant positive correlation was observed between p63 and E2A protein expression $(R=0.908 ; p<0.0001)$. In addition, clinical analysis of Kaplan-Meier dataset showed that LUSC patients with high expression of E2A exhibited poor overall survival (OS) (Fig. 4E). Together, these results indicate that E47 is positively correlated with p63 in SCC and that increased E47 expression is associated with poor clinical outcomes of SCC patients.

\section{Discussion}

In recent years, targeted therapy to treat adenocarcinoma has greatly improved patient outcomes. However, little progress has been made on targeted therapy for squamous cell carcinoma (SCC). It is urgently needed to decipher mechanisms and identify drug targets involved in the development of SCC.

There are evidently unique genomic alterations in adenocarcinoma (ADC) and SCC. One of the most differentially expressed genes between ADC and SCC is p $63^{47}$. p63 is often overexpressed in SCC and is a wellknown marker of squamous differentiation, whereas p63 is little expressed in advanced adenocarcinoma. In this study, we demonstrate the opposite roles of E47 on regulation of growth of ADC and SCC, in which $\triangle \mathrm{Np} 63 \alpha$ serves as a key mediator. Most significantly, E47 can directly bind to the $\triangle \mathrm{Np} 63 \alpha$ promoter in SCC $\mathrm{H} 292$ cells, but not in ADC A549 cells, consistent with the observations that A549 cells express very little $\Delta \mathrm{Np} 63 \alpha$ and that E47 is unable to transactivate $\Delta \mathrm{Np} 63 \alpha$ in A549 cells. The molecular mechanisms are not clear, but it is plausible that there may be lack of transcription-cofactor(s) required for E47 DNA-binding to the $\triangle \mathrm{Np} 63 \alpha$ promoter in A549 cells or that the epigenic landscape at the 
chromatin state (histone acetylation/methylation) at $\triangle \mathrm{Np} 63$ promoter is fundamentally different in ADC and SCC. Notably, our study is in line with the observations that the increased p63 expression promotes progressive transdifferentiation of lung ADC to SCC in mouse models $^{48,49}$. Therefore, we propose that the expression of $\triangle \mathrm{Np} 63 \alpha$ is a critical intrinsic link between ADC and SCC. It would be worthwhile to further investigate how the expression of $\Delta \mathrm{Np} 63 \alpha$ is lost during the development of ADC or expression of $\triangle \mathrm{Np} 63 \alpha$ is established during SCC development and whether E47 plays a role in transdifferentiation of lung ADC to SCC.

p63 plays an important role in regulation of a variety of biological processes, including embryonic development, differentiation, tumor growth, aging, tumor metastasis, and tumorigenesis ${ }^{23}$. It has been shown that p63 gene expression can be upregulated by several transcriptional factors. Upon keratinocyte differentiation, transcriptional factors $\mathrm{C} / \mathrm{EBP} \alpha$ and DEC1 can target $\triangle \mathrm{Np63}$ promoter to increase $\Delta \mathrm{Np} 63 \alpha$ transcription $^{50}$. Oct4 can target p63 promoter and stimulates p63 to promote cell transformation $^{51}$. In addition, WT1 can bind to the p63 promoters and activate its expression to promote cell proliferation $^{52}$. We have recently shown that FOXO3a can also directly transactivate $\Delta \mathrm{Np} 63 \alpha$ during cancer metas$\operatorname{tasis}^{25}$. In this study, we have identified that E47 is a novel direct transcription factor of $\Delta \mathrm{Np} 63$. E47 can bind to $\Delta$ Np63 promoter and increase its expression, thereby regulating expression of $\Delta \mathrm{Np} 63 \alpha$ targeted genes involved in cell proliferation, including cyclins D1/E1 and p21. We demonstrate that $\Delta \mathrm{Np} 63 \alpha$ protein is a critical target of E47 in promoting cell proliferation and tumor growth in squamous cell carcinoma.

Importantly, clinical validation of human lung SCC and esophagus SCC samples reveals a significant and positive correlation between p63 and E47, and that lung SCC patients with high E47 expression show poor overall survival. Therefore, targeting E47- $\Delta \mathrm{Np} 63 \alpha$ axis may be a potential therapeutic strategy for treatment of squamous cell carcinoma.

\section{Materials and methods \\ Cell culture}

Human lung cancer cells H292, esophageal squamous cell carcinoma cells KYSE150, head and neck squamous carcinoma cells FaDu, lung adenocarcinoma cells H1299/ A549, and human embryonic kidney cells HEK293T were obtained from the American Type Culture Collection (ATCC). H292 and KYSE150 cells were grown in RPMI1640 medium (Invitrogen, Carlsbad, CA, USA), supplemented with 10\% FBS (Hyclone, Logan, UT, USA). FaDu, H1299, A549, and HEK293T cells were grown in DMEM medium (Invitrogen, Carlsbad, CA, USA), supplemented with 10\% FBS (Hyclone, Logan, UT, USA). All cells were grown in the medium containing $1 \%$ penicillin G/streptomycin sulfate (GIBCO, Rockville, MD, USA) and were incubated at $37^{\circ} \mathrm{C}$ in a humidified incubator under $5 \%$ $\mathrm{CO}_{2}$. Authentication of cells was verified by short tandem repeat DNA profiling.

\section{Plasmids and lentiviral infection}

Human E47 cDNA was cloned into the plvx-puro lentiviral vector. Human $\triangle \mathrm{Np} 63$ promotor $(-2000 \sim-1)$ was cloned into the GLuc-ON promoter reporter vector. $\mathrm{E} 47^{\mathrm{A} 592 \mathrm{~N} / 1596 \mathrm{D}}$ and $\triangle \mathrm{Np} 63$ promotor deletion P2 site $(\Delta-1665 \sim-1656)$ were generated by KOD-PlusMutagenesis kit (SMK-101, Toyobo Osaka). A pLKO.1puromysci lentiviral vector was used to generate short hairpin RNAs (shRNAs) targeting GFP, E2A, or p63. Specific oligos of shRNA are used as follows. shGFP: 5'GAAGCAGCACGACTTCTTC-3'; shE2A: 5'-AGCAGCC TCTCTTCATCC-3'; shp63: 5'-GAGTGGAATGACTT CAACTTT- $3^{\prime}$. All the constructs were confirmed by direct DNA sequencing. Recombinant lentiviruses were amplified in HEK293T cells as described ${ }^{53}$.

\section{Western blotting analysis}

Cells were lysed in the EBC250 lysis buffer $(250 \mathrm{mM}$ $\mathrm{NaCl}, 25 \mathrm{mM}$ Tris $\mathrm{pH} 8.0,0.5 \%$ Nonidet $\mathrm{p}-40$, and supplemented with $50 \mathrm{mM} \mathrm{NaF}, 20 \mu \mathrm{g} / \mathrm{mL}$ aprotinin, $1 \mathrm{mM}$ phenylmethylsulfonyl fluoride, and $2 \mu \mathrm{g} / \mathrm{mL}$ leupeptin). Western blotting analysis were performed as described ${ }^{54}$. Antibody specific for E2A (sc-133074) was purchased from Santa Cruz Biotechnology (Burlingame CA, USA). Antibodies specific for p63 (CY5659), p21 (CY5543), and GAPDH (AB0037) were purchased from Abways (Shanghai, China). Antibodies specific for CyclinE1 (3327) and CyclinD1 (2261) were purchased from Epitomics (Burlingame CA, USA). GAPDH was used as loading control.

\section{mRNA isolation and quantitative RT-PCR}

Total mRNA was extracted using RNA isolation Kit (macherey-nagel) (Düren, Germany) according to the manufacturer's instructions, and $1 \mu \mathrm{g}$ RNA template was reverse-transcribed into cDNA by RT-PCR Quick master mix (TOYOBO, Osaka, Japan). cDNA was amplified using SYBR Green Master Mix (Bio-Rad, Hercules CA, USA) by using the primers: E47 Q-PCR primer: Forward: $5^{\prime}-\mathrm{G}$ AGGAGAAAGACCTGAGGGACC- $3^{\prime}$ and Reverse: $5^{\prime}$-A CCTGACACCTTTTCCTCTTCTC-3'; $\triangle$ Np63 Q-PCR primer: Forward: 5'-GAAAACAATGCCCAGACTCAA-3' and Reverse: $5^{\prime}$-TGCGCGTGGTCTGTGTTA-3'; CCNE1 Q-PCR primer: Forward 5'- TGTGTCCTGGATGTTGAC TGCC-3' and Reverse: 5'- CTCTATGTCGCACCACTG ATACC-3'; CCND1 Q-PCR primer: Forward 5'- TCTA CACCGACAACTCCATCCG-3' ${ }^{\prime}$ and Reverse: $5^{\prime}$ - TCT GGCATTTTGGAGAGGAAGTG-3'; CDKNIA Q-PCR 
primer: Forward 5' - AGGTGGACCTGGAGACTCTCA G-3' and Reverse: $5^{\prime}$ - TCCTCTTGGAGAAGATCAGC CG-3'; GAPDH Q-PCR primer: Forward 5'-GGGGAGCC AAAAAGGGTCATCATCT- $3^{\prime}$ and Reverse: 5'-GAGG GGCCATCCACAGTCTTCT-3'. Data are expressed as $\Delta \triangle \mathrm{Ct}$ (fold change).

\section{MTS assay and colony formation assay}

The MTS assay and colony formation assay were used to measure the ability of cell proliferation. For MTS assay, briefly, $1.5 \times 10^{3}$ cells/well (H292 or A549) or $1 \times 10^{3}$ cells/well (FaDu, KYSE150 or H1299) were seeded into in 96-well plates and incubated overnight. $10 \mu \mathrm{L}$ of MTS (G5421, Promega Corporation, Madison, WI, USA) were added to each well, and then cells were incubated for $1.5 \mathrm{~h}$ at $37^{\circ} \mathrm{C}$. The absorption was detected at a wavelength of $490 \mathrm{~nm}$ using Varioskan Flash (Thermo, Carlsbad CA, USA).

For colony formation assay, cells were seeded into 6-well plates with a density of 500 per well, with medium changed every 3 days. After culture for 7-9 days, the colonies were fixed with methyl alcohol and stained with $0.1 \%$ crystal violet for $20 \mathrm{~min}$, and the number of colonies was counted under the microscope (Nikon, Japan).

\section{Luciferase reporter assay}

Luciferase reporter assays were performed with SecretePair Dual Luminescence Assay Kit (GeneCopoeia, USA) according to the manufacturer's instructions. Briefly, HEK293T cells were co-transfected with $100 \mathrm{ng}$ of $\triangle$ Np63-Gluc-SEAP reporter and an indicated dose of E47 expressing plasmid for $36 \mathrm{~h}$. And then cell culture medium were collected and $\triangle \mathrm{Np} 63$-Gluc and SEAP activities were measured. The $\Delta \mathrm{Np} 63-\mathrm{Gluc}$ activity was normalized to SEAP activity.

\section{BrdU and PI staining assay}

For BrdU staining, briefly, cells were labeled with $10 \mu \mathrm{M}$ BrdU (B9285, Sigma-Aldrich, St. Louis, USA) for $1 \mathrm{~h}$. Cells were collected and fixed in fixation buffer (4\% formaldehyde with $0.1 \%$ Triton $\mathrm{X}-100$ in $1 \times \mathrm{PBS}$ ) for $15 \mathrm{~min}$ at room temperature. Cells were then washed twice in flow cytometry staining buffer before the treatment of DNase I at $37^{\circ} \mathrm{C}$ for $1 \mathrm{~h}$. Cells were then incubated with $5 \mu \mathrm{L}$ FITC-conjugated anti-BrdU (364103, Biolegend, San Diego, CA, USA) or isotype control (400137, Biolegend, San Diego, CA, USA) antibody for $30 \mathrm{~min}$ at room temperature protected from light. The samples were then analyzed using flow cytometer (Beckman Coulter). For PI staining, cells were collected and fixed in $70 \%$ ethanol at $-20^{\circ} \mathrm{C}$ before the treatment with RNase A and propidium iodide (PI) for $30 \mathrm{~min}$. The cell cycle analyses were performed by using Flow Cytometer (Beckman Coulter).

\section{ChIP assay}

ChIP assays were performed in $\mathrm{H} 292$ or A549 cells with ChIP-IT Kit (Active Motif, Carlsbad, CA, USA) using antibodies specific for E2A (sc-133074, Santa Cruz, Burlingame CA, USA) or normal mouse IgG (Invitrogen, Carlsbad CA, USA), according to the manufacturer's instructions. The primers for the $\triangle \mathrm{Np} 63$ promoter region using primers were as follows: P1 (nucleotides -2303 to -2167): Forward: 5'-ATTTCACAAATGAGGAATCTGA ATCC- $3^{\prime}$ and Reverse: $5^{\prime}$-TATCCACTATGGCTACTTG AGAACTT-3'; P2 (nucleotides -1730 to -1572 ): Forward: $5^{\prime}$-TGGATGATTTTCAGGATCATCCAAGT-3 ${ }^{\prime}$ and Reverse: $5^{\prime}$ - ACCTACTTACAAAATACAGTATAA TT-3'; P3 (nucleotides -885 to -734 ): Forward: 5'-AAGC CAATTGATATCTTATGCTTTA- $3^{\prime}$ and Reverse: $5^{\prime}$-CT ATTTGAATATTTCTTTTCTCTAT-3'; hSHP (nucleotides -474 to -120 ): Forward: $5^{\prime}$ - CCCCTGGCAGGAA TG-3' and Reverse: 5'- AGGTTAGGCAAACAAGC-3'; negative control (nucleotides -40 to +128 ): Forward: $5^{\prime}-$ TGCCTATAGTTGGGTATATATTA-3' and Reverse: $5^{\prime}$ AAGAAAGGACAGCAGCATTGAT- $3^{\prime}$. The $C_{\mathrm{t}}$ value of each ChIP sample was normalized to its corresponding input.

\section{In vivo tumor formation assay}

The sample size for animal studies was designed according to previous report ${ }^{55}$. 6-week-old female BALB/ $C$ nude mice were purchased (DOSSY EXPERIMENTAL ANIMALS CO.,LTD., Chengdu, China), randomly divided into four groups $(n=4 /$ group $)$ and housed under standard conditions. $1 \times 10^{6} \mathrm{H} 292$ stable cells in a $100 \mu \mathrm{L}$ PBS were subcutaneously injected into right flanks (four mice per group). Mice were monitored daily. Tumors were measured by a caliper every $2-3$ days and were calculated as Volume $=\left(\right.$ Length $\left.\times \mathrm{Width}^{2}\right) / 2$. No blinding test was used in assessing the outcome. When applicable, tumors were dissected, weighed, photographed, and fixed with $4 \%$ paraformaldehyde and embedded with paraffin for immunostaining analysis. Tumor volumes and weights were presented as means \pm SEM. All animal care and animal experiments in this study were performed in accordance with the institutional ethical guidelines and were approved by the institutional review board of Sichuan University.

\section{Immunohistological chemistry (IHC) and tissue microarray}

Tissue microarray slides of human squamous cell carcinoma specimens (HLug-S030PG02) were purchased (OUTDO, Shanghai, China). Antibodies specific against E2A (sc-133074, Santa Cruz, Burlingame CA, USA) and p63 (CY5659, Abways, Shanghai, China), Ki67 (9449, CST, USA) and cleaved caspase-3 (CC3) (9661, CST, USA) were used for immunohistochemistry (IHC) staining. Immunohistochemical assay was performed as 
described ${ }^{25}$. Slides were scanned by NanoZoomer (Hamamatsu, Japan). E2A and p63 images were analyzed by calculating the integrated optical density (IOD) using Image-Pro Plus 6.0 (Media Cybernetics, MA, USA), and the average optical density (AOD) was calculated using the formula: $\mathrm{AOD}=\mathrm{IOD} /$ Area as described ${ }^{56}$. Ki67 and CC3 images were analyzed by calculating the percentage of Ki67/CC3 positive tumor cell ${ }^{57}$.

\section{Clinical relevance analysis}

The mRNA levels of E2A in normal tissues or adenocarcinoma were analyzed using Oncomine dataset "Hao_Esophagus" or "Bhattacharjee_Lung". The mRNA levels of E2A in normal tissues or squamous cell carcinoma were analyzed using Oncomine dataset "Su_Esophagus" or "TCGA". Pearson correlation of E47 (E2A) and p63 expression was analyzed using Oncomine dataset "Su_Esophagus" or "Bild_Lung". Kaplan-Meier plots of overall survival (OS) of human squamous cell carcinoma patients stratified by the E2A mRNA expression levels were analyzed using the Kaplan-Meier survival datasets.

\section{Statistical analysis}

Data from cell culture were performed in three independent experiments. Data were presented as means \pm SD. The differences between two groups were performed using the two-tailed unpaired Student's $t$ test. The clinical data were compared after homogeneity tests. $p<0.05$ was considered statistically significant.

\section{Acknowledgements}

We thank members of Z.-X.X. laboratory for stimulating discussions during the course of study.

\section{Author contributions}

M.N. and Z.-X.X. conceived the project and performed the project planning; J.X., F. L., Y.G., R. G., L. D., M. F., and Y.Y. performed research; F. L. and H. C. contributed to clinical database analysis; M.N., Z.-X.X., and J.X. analyzed data and wrote the manuscript.

\section{Funding}

This work was supported by National Natural Science Foundation of China (81861148031, 31701242, 81830108, 81520108020) to Z.-X.X and M.N.; National Key R\&D Program of China (2018YFC2000100) to Z.-X.X.

\section{Data availability}

Data and resource are available from the corresponding authors.

\section{Ethics statement}

All animal care and animal experiments in this study were performed in accordance with the institutional ethical guidelines and were approved by the Animal Ethics Committee of the College of Life Sciences, Sichuan University, China.

\section{Conflict of interest}

The authors declare no competing interests.

\section{Publisher's note}

Springer Nature remains neutral with regard to jurisdictional claims in published maps and institutional affiliations.
Supplementary information The online version contains supplementary material available at https://doi.org/10.1038/s41419-021-03662-3.

Received: 4 December 2020 Revised: 17 March 2021 Accepted: 23 March 2021

Published online: 08 April 2021

\section{References}

1. Siegel, R. L., Miller, K. D. \& Jemal, A. Cancer Statistics, 2018. CA 68, 7-30 (2018).

2. Oser, M. G., Niederst, M. J., Sequist, L. V. \& Engelman, J. A. Transformation from non-small-cell lung cancer to small-cell lung cancer: molecular drivers and cells of origin. Lancet Oncol. 16, e165-e172 (2015).

3. Ferone, G. et al. SOX2 is the determining oncogenic switch in promoting lung squamous cell carcinoma from different cells of origin. Cancer Cell 30, 519-532 (2016).

4. Network, C. G. A. R. Comprehensive genomic characterization of squamous cell lung cancers The Cancer Genome Atlas Research Network (vol 489, pg 519, 2012). Nature 491, 288-288 (2012).

5. Travis, W. D. Pathology of lung cancer. Clin. Chest Med. 23, 65-6 (2002)

6. Li, Y. Y. et al. Genomic analysis of metastatic cutaneous squamous cell carcinoma. Clin. Cancer Res. 21, 1447-1456 (2015).

7. Pickering, C. R. et al. Mutational landscape of aggressive cutaneous squamous cell carcinoma. Clin. Cancer Res. 20, 6582-6592 (2014).

8. Cancer Genome Atlas, N. Comprehensive genomic characterization of head and neck squamous cell carcinomas. Nature 517, 576-582 (2015).

9. Herbst, R. S., Heymach, J. V. \& Lippman, S. M. Molecular origins of cancer: lung cancer. N. Engl. J. Med. 359, 1367-1380 (2008).

10. Kosaka, T. et al. Mutations of the epidermal growth factor receptor gene in lung cancer: biological and clinical implications. Cancer Res. 64, 8919-8923 (2004).

11. Ramalingam, S. S., Owonikoko, T. K. \& Khuri, F. R. Lung cancer: new biological insights and recent therapeutic advances. CA 61, 91-112 (2011).

12. Aguilar, E. J., Fraile, J. Z. \& Rodriguez, L. P. A. Necitumumab: a new option for first-line treatment of squamous cell lung cancer. Expert Opin. Drug Metab. Toxicol. 14, 765-772 (2018).

13. Levin, P. A. et al. Histologic transformation from adenocarcinoma to squamous cell carcinoma as a mechanism of resistance to EGFR inhibition. J. Thorac. Oncol. 10, E86-E88 (2015)

14. Pao, W. et al. Integration of molecular profiling into the lung cancer clinic. Clin. Cancer Res. 15, 5317-5322 (2009).

15. Hata, A. et al. A low DNA methylation epigenotype in lung squamous cell carcinoma and its association with idiopathic pulmonary fibrosis and poorer prognosis. Int. J. Cancer 146, 388-399 (2020).

16. Kawase, A. et al. Differences between squamous cell carcinoma and adenocarcinoma of the lung: are adenocarcinoma and squamous cell carcinoma prognostically equal? Jpn. J. Clin. Oncol. 42, 189-195 (2012).

17. Argyris, P. P., Slama, Z. M., Ross, K. F., Khammanivong, A. \& Herzberg, M. C Calprotectin and the initiation and progression of head and neck cancer. J. Dent. Res. 97, 674-682 (2018).

18. Grau, C. et al. Sino-nasal cancer in Denmark 1982-1991 - a nationwide survey. Acta Oncol. 40, 19-23 (2001).

19. Su, C. et al. Predictive and prognostic effect of CD133 and cancer-testis antigens in stage Ib-IIIA non-small cell lung cancer. Int. J. Clin. Exp. Pathol. 8, 5509-5518 (2015).

20. Campbell, J. D. et al. Genomic, pathway network, and immunologic features distinguishing squamous carcinomas. Cell Rep. 23, 194-19 (2018).

21. Mangiulli, M. et al. Identification and functional characterization of two new transcriptional variants of the human p63 gene. Nucleic Acids Res. 37, 6092-6104 (2009).

22. Yang, A. N. et al. p63, a p53 homolog at 3q27-29, encodes multiple products with transactivating, death-inducing, and dominant-negative activities. Mol. Cell 2, 305-316 (1998).

23. Sethi, I. et al. A global analysis of the complex landscape of isoforms and regulatory networks of p63 in human cells and tissues. BMC Genom. 16, 584 (2015).

24. Candi, E. et al. Differential roles of p63 isoforms in epidermal development: selective genetic complementation in p63 null mice. Cell Death Differ. 13 1037-1047 (2006). 
25. Hu, L. et al. DeltaNp63alpha is a common inhibitory target in oncogenic PI3K Ras/Her2-induced cell motility and tumor metastasis. Proc. Natl Acad. Sci. USA 114, E3964-E3973 (2017).

26. Leonard, M. K. et al. DeltaNp63alpha regulates keratinocyte proliferation by controlling PTEN expression and localization. Cell Death Differ. 18, 1924-1933 (2011).

27. Wang, L., Xia, W., Chen, H. \& Xiao, Z. X. DeltaNp63alpha modulates phosphorylation of p38 MAP kinase in regulation of cell cycle progression and cell growth. Biochem. Biophys. Res. Commun. 509, 784-789 (2019).

28. DeYoung, M. P. et al. Tumor-specific p73 up-regulation mediates p63 dependence in squamous cell carcinoma. Cancer Res. 66, 9362-9368 (2006).

29. Schavolt, K. L. \& Pietenpol, J. A. p53 and Delta Np63 alpha differentially bind and regulate target genes involved in cell cycle arrest, DNA repair and apoptosis. Oncogene 26, 6125-6132 (2007).

30. Westfall, M. D., Mays, D. J., Sniezek, J. C. \& Pietenpol, J. A. The delta Np63 alpha phosphoprotein binds the p21 and 14-3-3 sigma promoters in vivo and has transcriptional repressor activity that is reduced by Hay-Wells syndromederived mutations. Mol. Cell Biol. 23, 2264-2276 (2003).

31. Ramsey, M. R., He, L., Forster, N., Ory, B. \& Ellisen, L. W. Physical association of HDAC1 and HDAC2 with p63 mediates transcriptional repression and tumor maintenance in squamous cell carcinoma. Cancer Res. 71, 4373-4379 (2011).

32. Zhang, Y., Yan, W. \& Chen, X. P63 regulates tubular formation via epithelial-tomesenchymal transition. Oncogene 33, 1548-1557 (2014).

33. Lefkimmiatis, K., Caratozzolo, M. F., Merlo, P., D'Erchia, A. M., Navarro, B. \& Levrero, M. et al. p73 and p63 sustain cellular growth by transcriptional activation of cell cycle progression genes. Cancer Res. 69, 8563-8571 (2009).

34. Ye, S., Lee, K. B., Park, M. H., Lee, J. S. \& Kim, S. M. p63 regulates growth of esophageal squamous carcinoma cells via the Akt signaling pathway. Int. J. Oncol. 44, 2153-2159 (2014).

35. Yang, A. \& McKeon, F. P63 and P73: P53 mimics, menaces and more. Nat. Rev. Mol. Cell Biol. 1, 199-207 (2000).

36. Yamazaki, T. et al. TCF3 alternative splicing controlled by hnRNP H/F regulates E-cadherin expression and hESC pluripotency. Gene Dev. 32, 1161-1174 (2018).

37. Murre, C. et al. Interactions between heterologous helix-loop-helix proteins generate complexes that bind specifically to a common DNA-sequence. Cell 58, 537-544 (1989).

38. Schwartz, R., Engel, I., Fallahi-Sichani, M., Petrie, H. T. \& Murre, C. Gene expression patterns define novel roles for E47 in cell cycle progression, cytokine-mediated signaling, and T lineage development. Proc. Natl Acad. Sci. USA 103, 9976-9981 (2006).

39. Shen, C. P. \& Kadesch, T. B-cell-specific DNA-binding by an E47 homodimer. Mol. Cell Biol. 15, 4518-4524 (1995).

40. Voronova, A. F. \& Lee, F. The E2a and Tal-1 helix-loop-helix proteins associate in-vivo and are modulated by Id proteins during interleukin-6-induced myeloid differentiation. Proc. Natl Acad. Sci. USA 91, 5952-5956 (1994).
41. Kim, S. et al. The basic helix-loop-helix transcription factor E47 reprograms human pancreatic cancer cells to a quiescent acinar state with reduced tumorigenic potential. Pancreas 44, 718-727 (2015).

42. Wilson, J. W., Deed, R. W., Inoue, T., Balzi, M., Becciolini, A. \& Faraoni, P. et al. Expression of Id helix-loop-helix proteins in colorectal adenocarcinoma correlates with p53 expression and mitotic index. Cancer Res. 61, 8803-8810 (2001).

43. Scully, K. M. et al. E47 governs the MYC-CDKN1B/p27(KIP1)-RB network to growth arrest PDA cells independent of CDKN2A/p16(INK4A) and wild-type p53. Cell Mol. Gastroenterol. Hepatol. 6, 181-198 (2018).

44. Sun, R. et al. Human calcium/calmodulin-dependent serine protein kinase regulates the expression of p21 via the E2A transcription factor. Biochem. J. 419, 457-466 (2009).

45. Pfurr, S. et al. The E2A splice variant E47 regulates the differentiation of projection neurons via p57(KIP2) during cortical development. Development 144, 3917-3931 (2017).

46. Kim, H. J., Kim, J. Y., Park, Y. Y. \& Choi, H. S. Synergistic activation of the human orphan nuclear receptor SHP gene promoter by basic helix-loop-helix protein E2A and orphan nuclear receptor SF-1. Nucleic Acids Res. 31, 6860-6872 (2003).

47. Conde, E. et al. The use of P63 immunohistochemistry for the identification of squamous cell carcinoma of the lung. PLOS ONE 5, e12209 (2010).

48. Zhang, W. et al. YAP promotes malignant progression of Lkb1-deficient lung adenocarcinoma through downstream regulation of survivin. Cancer Res. 75, 4450-4457 (2015).

49. Ji, H. et al. LKB1 modulates lung cancer differentiation and metastasis. Nature 448, 807-810 (2007).

50. Patrick, B. A. \& Jaiswal, A. K. Stress-induced NQO1 controls stability of C/ EBPalpha against $20 \mathrm{~S}$ proteasomal degradation to regulate p63 expression with implications in protection against chemical-induced skin cancer. Oncogene 31, 4362-4371 (2012).

51. Ng, W. L. et al. OCT4 as a target of miR-34a stimulates p63 but inhibits p53 to promote human cell transformation. Cell Death Dis. 5, e1024 (2014).

52. Li, X. R. et al. Wilms' tumor gene 1 regulates p63 and promotes cell proliferation in squamous cell carcinoma of the head and neck. BMC Cancer 15 342 (2015).

53. Yi, Y. et al. Transcriptional suppression of AMPK alpha 1 promotes breast cancer metastasis upon oncogene activation. Proc. Natl Acad. Sci. USA 117, 8013-8021 (2020).

54. Sun, S. et al. Hotspot mutant p53-R273H inhibits KLF6 expression to promote cell migration and tumor metastasis. Cell Death Dis. 11, 595 (2020).

55. Charan, J. \& Kantharia, N. D. How to calculate sample size in animal studies? J. Pharm. Pharmacother. 4, 303-306 (2013).

56. Tilley, W. D. et al. Detection of discrete androgen receptor epitopes in prostate-cancer by immunostaining - measurement by color video imageanalysis. Cancer Res. 54, 4096-4102 (1994).

57. Laurinavicius, A. et al. A methodology to ensure and improve accuracy of Ki67 labelling index estimation by automated digital image analysis in breast cancer tissue. Breast Cancer Res. 16, R35 (2014). 